\title{
Theory of microphase segregation in the melts of copolymers with dendritically branched, bottlebrush or cycled blocks.
}

\author{
Ekaterina B. Zhulina ${ }^{1}$, \\ Sergei S.Sheiko ${ }^{2}$, Oleg V.Borisov ${ }^{1,3}$ \\ ${ }^{1}$ Institute of Macromolecular Compounds \\ of the Russian Academy of Sciences, \\ 199004 St.Petersburg, Russia \\ 2 Department of Chemistry, University of North Carolina \\ at Chapel Hill, 27599, USA \\ ${ }^{3}$ Institut des Sciences Analytiques et de Physico-Chimie pour \\ l'Environnement et les Matériaux, UMR 5254 CNRS UPPA, \\ 64053 Pau, France
}

July 29, 2019

email: oleg.borisov@univ-pau.fr

Supporting Information

\section{Strong stretching self-consistent field (SS-SCF) theory of linear polymer chain brushes.}

The self-consistent field strong stretching (SS-SCF) theory of dry (solventfree) polymer brushes were formulated originally by Semenov ${ }^{1}$ in the analysis of superstructures formed in microsegregated melts of diblock copolymers. Later this theory was extended, ${ }^{2}$ to polymer brushes immersed in low molecular weight solvent of arbitrary thermodynamic quality.

Consider a solvent-free brush of linear macromolecules end-attached to a planar surface. The total number of monomer units in one macromolecule is $N$, the chains are flexible with Kuhn segment length on the order of monomer 
size $a$. Macromolecules are tethered to the surface with grafting density $\sigma$, and exhibit the Gaussian elasticity on all length scales. The thickness $H$ of a solvent-free brush is given by $H=N a^{3} \sigma$.

Within the strong stretching (called also quasi-classical) approximation the set of conformations for each of the brush-forming chains is mapped to the set of trajectories that specify position $z(s)$ of monomer unit with ranking number $s$ above the grafting surface. By introducing the stretching function $E=d z(s) / d s$, which is directly related in the linear elasticity regime to the chain local tension, $T(z)=3 k_{B} T E(z) / a^{2}$, the free energy per chain in the dry brush is presented as

$$
\frac{F}{k_{B} T}=\frac{3}{2 a^{2}} \int_{0}^{H} g\left(z^{\prime}\right) d z^{\prime} \int_{0}^{z^{\prime}} E\left(z^{\prime}, z\right) d z
$$

where $E\left(z, z^{\prime}\right)$ is local stretching of the chain at distance $z$ from the surface provided that the free end of the chain is at $z^{\prime} \geq z, H$ is the brush thickness and function $g(z)$ describes distribution of the free ends of the chains in the direction perpendicular to the grafting surface. If function $g(z)$ is normalized as

$$
\int_{0}^{H} g(z) d z=1
$$

then the volume fraction of monomer units at distance $z$ from the surface is expressed as

$$
\phi(z)=a^{3} \sigma \int_{z}^{H} \frac{g\left(z^{\prime}\right)}{E\left(z^{\prime}, z\right)} d z^{\prime}
$$

and $\phi(z) \equiv$ in the dry (solvent-free) brush.

Minimization of the free energy with the account of the space-filling condition $\phi(z) \equiv 1$, and conservation of the number of monomer units in the chain irrespective of location of its end segment,

$$
\int_{0}^{z^{\prime}} \frac{d z}{E\left(z^{\prime}, z\right)}=N
$$

comes down to minimization of the functional

$$
\begin{gathered}
\Phi=\frac{3}{2 a^{2}} \int_{0}^{H} g\left(z^{\prime}\right) d z^{\prime} \int_{0}^{z^{\prime}} E\left(z^{\prime}, z\right) d z+ \\
\int_{0}^{H} \lambda_{1}\left(z^{\prime}\right) \int_{0}^{z^{\prime}} \frac{d z}{E\left(z^{\prime}, z\right)}+\int_{0}^{H} \lambda_{2}(z) \int_{z}^{H} \frac{g\left(z^{\prime}\right)}{E\left(z^{\prime}, z\right)} d z^{\prime}
\end{gathered}
$$


in which $\lambda_{1}(z)$ and $\lambda_{2}(z)$ constitute the Lagrangian multipliers. By varying the functional in eq 5 with respect to $E\left(z, z^{\prime}\right)$ and applying the condition of vanishing tension at the free ends of the chains,

$$
E\left(z^{\prime}, z^{\prime}\right)=0
$$

one obtains

$$
E^{2}\left(z^{\prime}, z\right)=\frac{2 a^{2}}{3}\left(U\left(z^{\prime}\right)-U(z)\right)
$$

with

$$
U(z) \equiv-\lambda_{2}(z)
$$

as (measured in $k_{B} T$ units) self-consistent Lagrangian potential assuring the space filling condition. The explicit expression for $U(z)$ is obtained by applying the condition of conservation of monomer units in each chain, that is, by substituting eq 6 into eq 4 and solving eq 4 that leads to

$$
\begin{aligned}
U(z) & =-\frac{3 \pi^{2}}{8 N^{2} a^{2}} z^{2}+\text { const } \\
E\left(z^{\prime}, z\right) & =\sqrt{\frac{2 a^{2}}{3}\left(U\left(z^{\prime}\right)-U(z)\right),}
\end{aligned}
$$

and, finally,

$$
E\left(z^{\prime}, z\right)=\frac{\pi}{2 N} \sqrt{z^{\prime 2}-z^{2}}
$$

Remarkably eq 8 applies not only in the case of dry brushes, but also for brushes immersed in a solvent (with $\phi(z)<1$ ). In the latter case, the dimensionless self-consistent potential is still given by eq 7 , and is linked to the density $f(\phi(z))$ of the free energy of monomer-monomer interactions as

$$
U(z)=\frac{\delta f(\phi(z))}{k_{B} T \delta \phi(z)}
$$

\section{Generalization of strong stretching self-consistent field (SS-SCF) theory for dendron brushes}

The described above SS-SCF approach was generalized in refs, ${ }^{34}$ for brushes formed by regular dendrons and later in $\mathrm{refs}^{5-7}$ for brushes formed by branched macromolecules with arbitrary tree-like architecture or containing macrocycles. $^{8}$

Consider now a solvent-free brush of branched (tree-like) macromolecules attached by the root segments to a planar surface. The total number of monomer units in the macromolecule is $N \gg 1$. Macromolecules are tethered 
to the surface with grafting density $\sigma$. The thickness of a solvent-free brush, $H=N a^{3} \sigma$, does not depend on the chain architecture.

As long as (i) the brush-forming branched macromolecules are monodisperse, (ii) all linear segments of these macromolecules exhibit Gaussian elasticity on all length scales and (iii) the distributions of the end-segments of free branches exhibit no dead zones (depleted of the end-segments), the selfconsistent molecular potential in the brush has a parabolic form, similar to that given by eq 7 ,

$$
U(z)=-\frac{3}{2 a^{2}} \kappa^{2} z^{2}+\text { const }
$$

with prefactor $\kappa$ depending on the total number $N$ of monomers units, and specific macromolecular topology (connectivity of the spacers). Obviously, for linear chains $\kappa_{\text {lin }}=\pi / 2 N$.

The stretching function for a free branch with the end position $z_{e}$ still obeys eq 8 ,

$$
E_{\text {free }}\left(z_{e}, z\right)=\kappa \sqrt{z_{e}^{2}-z^{2}}
$$

while the stretching functions for strands (spacers), connecting the branching points, have the form

$$
E_{\text {spacer }}\left(\lambda_{i j}, z\right)=\kappa \sqrt{\lambda_{i j}^{2}-z^{2}},
$$

with subscripts $i j$ specifying location of the particular spacer in the tree-like structure (e.g., the $j$-th spacer in the $i$-th branching generation).

For each architecture (topology) of the macromolecule, the topological coefficient $\kappa$ and the set of constants $\{\lambda i j\}$ can be found from the following requirements: (i) balance of the elastic tensions in each branching point, and (ii) conservation of the number of monomer units in each of the spacers (and thereby in equivalent paths of the tree-like macromolecule).

\section{Example of derivation of the topological coefficient}

Below we illustrate the outlined calculation scheme by deriving an equation for the topological coefficient $\kappa$ in a brush formed by asymmetric dendrons of the second generation, depicted in Figure S1.

In Figure S1, the stem segment of the tethered macromolecule has $n_{0}$ monomer units, and the first branching point is located at height $z_{0}$. The first generation comprises two branches with number of monomer units $n_{2}$ each, and one branch with number of monomer units $n_{1}$. The two branching points at the ends of $n_{2}$-segments are located at height $z_{2}$, and the branching point of $n_{1}$-segment at height $z_{1}$. The latter branching point gives rise to $q_{1}$ of 


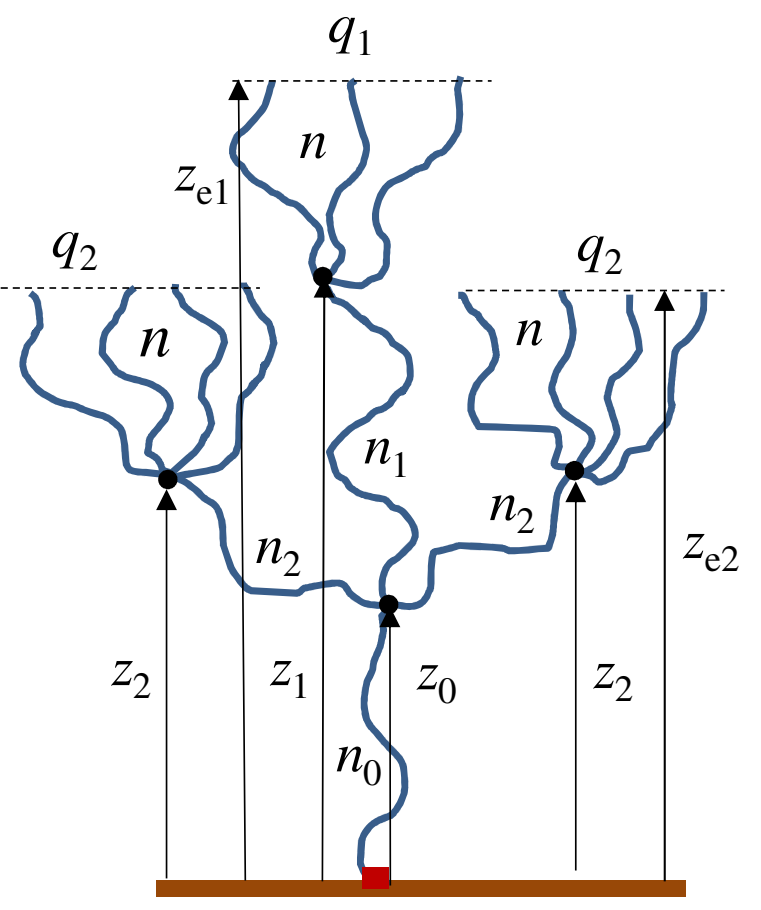

Figure S1. Schematics of asymmetric dendron with two generations 
free branches (with number of monomer units $n$ each) in the second generation, while two other branching points produce $q_{2}$ similar free branches each. The positions of free end-points are indicated as $z_{e 1}$ and $z_{e 2}$, respectively. The asymmetry of this particular dendron is traced to the different lengths of branches in the first generation $\left(n_{1} \neq n_{2}\right)$, and the different branching activities in the second generation $\left(q_{1} \neq q_{2}\right)$. A fully symmetric dendron with branching activity 3 (i.e., with three branches originating from the first branching point) is characterized by $n_{0}=n_{1}=n_{2}=n$, and $q_{1}=q_{2}=3$.

As mentioned above, in a parabolic potential, the stretching functions of the free branches, $E_{\text {free }, 1}$ and $E_{\text {free, } 2}$, are specified as

$$
\begin{array}{ll}
E_{\text {free }, 1}\left(z_{e 1}, z\right)=\sqrt{\frac{2 a^{2}}{3}\left[U\left(z_{e 1}\right)-U(z)\right]}=\kappa \sqrt{z_{e 1}^{2}-z^{2}} & z_{1} \leq z \leq z_{e 1} \\
E_{\text {free }, 2}\left(z_{e 2}, z\right)=\sqrt{\frac{2 a^{2}}{3}\left[U\left(z_{e 2}\right)-U(z)\right]}=\kappa \sqrt{z_{e 2}^{2}-z^{2}} & z_{2} \leq z \leq z_{e 2}
\end{array}
$$

They ensure vanishing tension at the free ends of the branches, $z=z_{e 1}$ and $z=z_{e 2}$. By using the conservation conditions

$$
\int_{z_{1}}^{z_{e 1}} \frac{d z}{E_{\text {free }, 1}\left(z_{e 1,}, z\right)}=\int_{z_{2}}^{z_{e 2}} \frac{d z}{E_{\text {free }, 2}\left(z_{e 2,}, z\right)}=n,
$$

and performing the corresponding integrations, we find the relationship between positions of the free ends and the respective branching points,

$$
\frac{z_{1 e}}{z_{1}}=\frac{z_{2 e}}{z_{2}}=\frac{1}{\cos (\kappa n)}
$$

The stretching function of the stem is given by

$$
E_{0}=\kappa \sqrt{\lambda_{0}^{2}-z^{2}}=\kappa \sqrt{\frac{z_{0}^{2}}{\sin ^{2}\left(\kappa n_{0}\right)}-z^{2}},
$$

in which constant $\lambda_{0}$ is found from the normalization condition

$$
\int_{0}^{z_{0}} \frac{d z}{E_{0}\left(\lambda_{0}, z\right)}=n_{0}
$$

The stretching functions of the branches in the first generation $\left(E_{2}\right.$ and $\left.E_{1}\right)$ are given by

$$
E_{1}=\kappa \sqrt{\lambda_{1}^{2}-z^{2}} ; \quad E_{2}=\kappa \sqrt{\lambda_{2}^{2}-z^{2}}
$$


with indefinite constants $\lambda_{1}$ and $\lambda_{2}$ specified from balancing the elastic tensions in the branching points of the second generation. That is,

$$
E_{1}\left(z_{1}\right)=q_{1} E_{\text {free }, 1}\left(z_{1}\right) ; \quad E_{2}\left(z_{2}\right)=q_{2} E_{\text {free }, 2}\left(z_{2}\right),
$$

to give

$$
\left.\lambda_{1}^{2}=z_{1}^{2}\left[1+q_{1}^{2} \tan ^{2}(\kappa n)\right] \quad \text { and } \quad \lambda_{2}^{2}=z_{2}^{2}\left[1+q_{2}^{2} \tan \kappa n\right)\right]
$$

The conservation conditions for the chain segments in the first generation,

$$
\int_{z_{0}}^{z_{1}} \frac{d z}{E_{1}\left(\lambda_{1}, z\right)}=n_{1} ; \quad \int_{z_{0}}^{z_{2}} \frac{d z}{E_{2}\left(\lambda_{2}, z\right)}=n_{2}
$$

lead to the following expressions:

$$
\lambda_{1}^{2}=\frac{z_{1}^{2}-2 z_{1} z_{0} \cos \left(\kappa n_{1}\right)+z_{0}^{2}}{\sin ^{2}\left(\kappa n_{1}\right)} ; \quad \lambda_{2}^{2}=\frac{z_{2}^{2}-2 z_{2} z_{0} \cos \left(\kappa n_{2}\right)+z_{0}^{2}}{\sin ^{2}\left(\kappa n_{2}\right)} .
$$

By eliminating $\lambda_{1}^{2}$ and $\lambda_{2}^{2}$ from eqs 21 and 23, we find ratios $z_{2} / z_{0}$ and $z_{1} / z_{0}$ as

$$
\begin{aligned}
& \frac{z_{2}}{z_{0}}=\frac{1}{\cos \left(\kappa n_{2}\right)-q_{2} \sin \left(\kappa n_{2}\right) \tan (\kappa n)}, \\
& \frac{z_{1}}{z_{0}}=\frac{1}{\cos \left(\kappa n_{1}\right)-q_{1} \sin \left(\kappa n_{1}\right) \tan (\kappa n)} .
\end{aligned}
$$

The force balance in the first branching point, $2 E_{2}\left(z_{0}\right)+E_{1}\left(z_{0}\right)=E_{0}\left(z_{0}\right)$, yields

$$
2 \sqrt{\lambda_{2}^{2}-z_{0}^{2}}+\sqrt{\lambda_{1}^{2}-z_{0}^{2}}=\frac{z_{0}}{\tan \left(\kappa n_{0}\right)} .
$$

By substituting $\lambda_{1}^{2}$ and $\lambda_{2}^{2}$ from eqs 23 in eq 26, one finds

$$
2 \frac{\left(z_{2} / z_{0}\right)-\cos \left(\kappa n_{2}\right)}{\sin \left(\kappa n_{2}\right)}+\frac{\left(z_{1} / z_{0}\right)-\cos \left(\kappa n_{1}\right)}{\sin \left(\kappa n_{1}\right)}=\frac{1}{\tan \left(\kappa n_{0}\right)},
$$

and, with the account of eqs 24 and 25, the final equation for topological coefficient $\kappa$ is formulated as

$$
\tan \left(\kappa n_{0}\right)\left[2 \frac{\tan \left(\kappa n_{2}\right)+q_{2} \tan (\kappa n)}{1-q_{2} \tan \left(k n_{2}\right) \tan (k n)}+\frac{\tan \left(\kappa n_{1}\right)+q_{1} \tan (\kappa n)}{1-q_{1} \tan \left(k n_{1}\right) \tan (k n)}\right]=1 .
$$

The minimal numerical solution of eq 27 provides the value of $\kappa$ as a function of the architectural parameters $\left\{n_{0}, n_{1}, n_{2}, n, q_{1}, q_{2}\right\}$. If the numbers of $n_{2^{-}}$ branches and $n_{1}$-branches in the first generation change from 2 to $q_{0,2}$ and from 1 to $q_{0,1}$, the modification of eq 27 is straightforward,

$$
\tan \left(\kappa n_{0}\right)\left[q_{0,2} \frac{\tan \left(\kappa n_{2}\right)+q_{2} \tan (\kappa n)}{1-q_{2} \tan \left(k n_{2}\right) \tan (k n)}+q_{0,1} \frac{\tan \left(\kappa n_{1}\right)+q_{1} \tan (\kappa n)}{1-q_{1} \tan \left(k n_{1}\right) \tan (k n)}\right]=1 .
$$


Eq 28 specifies the topological coefficient $\kappa$ for a family of dendrons comprising two generations. It is simplified in a few special cases. For example, for linear chain with total number $N=n_{0}+n_{1}+n$ of monomer units $\left(q_{0,2}=0\right.$, $\left.q_{0,1}=q_{1}=q_{2}=1\right)$, eq 28 reduces to $\tan \left(\kappa n_{0}\right) \tan \left(\kappa n_{1}+\kappa n\right)=1$. By using the equality, $\tan x+\tan y=\tan (x+y)(1-\tan x \tan y)$, and assigning $x=\kappa n_{0}$ and $y=\kappa n_{1}+\kappa n$, one finds $\tan \left(\kappa n_{0}+\kappa n_{1}+\kappa n\right)=\infty$ to produce the known result $^{1}$ for linear chains, $\kappa=\pi /(2 N)$. In the case of symmetric dendrons with $n_{0}=n_{1}=n_{2}=n$, and $q_{0,2}+q_{0,2}=q_{1}=q_{2}=q$, the minimal solution of eq 28 is given by $\kappa=n^{-1} \arctan [1 / \sqrt{q(q+2)}]$, the result obtained earlier in ref. ${ }^{7}$

The self-consistent potential $U(z)$ with the topological coefficient $k$ specified by eq 27 ensures the conservation of the total number $N$ of monomer units in the macromolecule depicted in Figure S1 (as well as the degrees of polymerization of all its branches) at any position of the end-points. In the presented framework, the positions of all branching points and of the free ends are linked to each other. By fixing, e.g., $z_{e 1}$, one finds $z_{1}$ via eq $16, z_{0}$ via eq $25, z_{2}$ via eq 24 , and $z_{e 2}$ via eq 16 .

Notably, the value of $\kappa$ does not depend on the grafting density $\sigma$ of the macromolecules, curvature of the grafting surface, or the solvent quality, and thereby holds in a variety of branched systems. At the same time, the solution of eq 27 ( $\kappa$-value) does not ensure the absence of dead zones depleted of the free ends, that may emerge in convex, and for certain architectural parameters of tethered macromolecules in planar brushes. For example, a decrease in the stem length $n_{0}$ could lead to the development of dead zone in a planar brush of $\psi$-polymers. ${ }^{9}$ A convenient tool to check for possible presence of the dead zones is the numerical SCF model of Scheutjens and Fleer (SF-SCF) which allows for quick determination of the parameter space eligible for the parabolic molecular potential..$^{8-10}$

\section{Topological ratios for selected architectures}

Using the outlined calculation scheme (executed above for a brush of dendrons comprising two generations), the values of topological coefficient $\kappa$ for symmetric and asymmetric dendrons of higher generations, starlike and comblike macromolecules, and macrocycle-containing polymers have been calculated in refs. ${ }^{5-8}$ Introduction of the topological ratios

$$
\eta=\kappa / \kappa_{\text {lin }}=\frac{2 N \kappa}{\pi}
$$

allows for direct comparison of the brush properties formed by the branched and linear macromolecules that have the same total number $N$ of monomer 
units. The topological ratio serves as a quantitative measure of the degree of branching of the brush-forming macromolecules: Evidently it is equal to unity for linear chains, while an increase in the degree of the chain branching leads to the increase in $\eta$ beyond unity.

Below we present the analytical expressions for topological ratio $\eta_{j}$ for regular dendrons of the $j$-th generation $(j=1,2,3)$, where all the spacers and free branches have the same length, and all the branching points have the same functionality.

Symmetric starlike polymers (dendrons of the first generation) with $q_{1}$ free branches of $n_{1}$ monomer units each, have the total number of monomer units $N_{1}=n_{1}\left(1+q_{1}\right)$, and:

$$
\begin{gathered}
\kappa_{1}=n_{1}^{-1} \arctan \left(q_{1}^{-1 / 2}\right), \\
\eta_{1}=\frac{2\left(q_{1}+1\right)}{\pi} \arctan \left(\frac{1}{\sqrt{q_{1}}}\right) \approx \frac{2}{\pi} \sqrt{q_{1}} \text { if } q_{1} \gg 1 .
\end{gathered}
$$

Symmetric dendrons of the second generation have total number $N_{2}=n_{2}(1+$ $\left.q_{2}+q_{2}^{2}\right)$ of monomer units, and:

$$
\begin{gathered}
\kappa_{2}=n_{2}^{-1} \arctan \left(1 / \sqrt{q_{2}\left(2+q_{2}\right)}\right), \\
\eta_{2}=\frac{2\left(q_{2}^{2}+q_{2}+1\right)}{\pi} \arctan \left(\frac{1}{\sqrt{q_{2}\left(2+q_{2}\right)}}\right) \approx \frac{2}{\pi} q_{2} \text { if } q_{2} \gg 1
\end{gathered}
$$

Symmetric dendrons of the third generation have the total number $N_{3}=$ $n_{3}\left(1+q_{3}+q_{3}^{2}+q_{3}^{3}\right)$ of monomer units, and:

$$
\begin{gathered}
\kappa_{3}=n_{3}^{-1} \cdot \arctan \sqrt{\frac{\left(q_{3}^{2}+2 q_{3}+3\right)-\sqrt{\left(q_{3}^{2}+2 q_{3}+3\right)^{2}-4}}{2 q_{3}}} \\
\eta_{3}=\frac{2\left(1+q_{3}+q_{3}^{2}+q_{3}^{3}\right)}{\pi} . \\
\arctan \sqrt{\frac{\left(q_{3}^{2}+2 q_{3}+3\right)-\sqrt{\left(q_{3}^{2}+2 q_{3}+3\right)^{2}-4}}{2 q_{3}}} \approx \frac{2}{\pi} q_{3}^{3 / 2} \text { if } q_{3} \gg 1
\end{gathered}
$$

Even though analytical expressions for the topological ratios for dendrons of higher generations are not available, one can conclude from eqs 29-31 that for regular dendrons of arbitrary generation $g$ the following asymptotic expression for the topological ratios applies at large branching functionality $q \gg 1$ :

$$
\eta \approx \frac{2}{\pi} q^{g / 2}
$$




\section{References}

[1] Semenov, A. N. Contribution to the Theory of Microphase Layering in Block-Copolymer Melts. Sov. Phys. JETP 1985, 61, 733-742.

[2] Zhulina, E.B.; Pryamitsyn, V.A.; Borisov, O.V. Structure and Conformational Transitions in Grafted Polymer Chain Layers: New Theory Polymer Science USSR 1989, 31 (1), 205-215.

[3] Pickett G. T. Classical Path Analysis of end-Grafted Dendrimers: Dendrimer Forest. Macromolecules 2001, 34, 8784-8791.

[4] Zook, T. C.; Pickett, G. T. Hollow-Core Dendrimers Revised. Physical review letters 2003, $90(1), 015502$.

[5] Polotsky, A. A.; Leermakers, F. A. M.; Zhulina, E. B.; Birshtein, T. M. On the Two-Population Structure of Brushes Made of Arm-Grafted Polymer Stars. Macromolecules 2012, 45, 7260-7273.

[6] Zhulina, E. B. ; Leermakers, F. A. M.; Borisov O.V. Ideal mixing in multicomponent brushes of branched macromolecules. Macromolecules 2015, 48 (23), 5614-5622.

[7] Zhulina, E.B.; Leermakers, F.A.M.; Borisov, O.V. Self-consistent Field Model of Brushes formed by Root-tethered Dendrons - Scientific and Technical Journal of Information Technologies, Mechanics and Optics 2015, 15 (3), 493-499.

[8] Zhulina, E. B. ; Leermakers, F. A. M.; Borisov O.V. Brushes of Cycled Macromolecules: Structure and Lubricating Properties. Macromolecules 2016, 49 (23), 8758-8767.

[9] Zhulina, E.B.; F.A.M. Leermakers, F.A.M.; Borisov, O.V. Theory of Brushes Formed by $\Psi$-shaped Macromolecules at Solid-Liquid Interfaces. Langmuir 2015, 31 (23), 6514-6522.

[10] Rud,O.V.; Polotsky, A.A.; Guillich, T.; Borisov, O.V.; Leermakers, F. A. M.; Textor, M.; Birshtein, T.M. Dendritic Spherical Polymer Brushes: Theory and Self-Consistent Field Modelling. Macromolecules 2013, 46, 4651-4662. 